\title{
Malestar psicológico determinado socialmente y abuso expresivo en varones
}

\author{
Fernando Bolaños Ceballos'
}

Artículo original

\section{SUMMARY}

The meanings of the abuses made by men to express discomfort were explored. A logic of accumulation of discomfort with social origin expressed in an act of abuse and mediated for a reason or conflict that justified the physical and/or verbal attack was described. Sometimes, the expression of discomfort was accompanied by guilt feelings after violent events. The expressive elements that allow abuses to partners and other family members are the beliefs about the superiority of men, the physical inferiority of women and children or the belief about their inferiority, the notion that wives are a property, the perception that relationships should last a lifetime, and beliefs about the use of violence in relationships. In terms of the intervention with this population, the study highlights the importance of considering the role of social determinants of distress from the macro level to the social programs and community interventions.

Key words: Social determination, psychological distress, men, family violence, abuse.

\section{RESUMEN}

El objetivo de este artículo es conocer y describir la experiencia subjetiva de hombres que asisten a un programa reeducativo para detener su violencia familiar, en torno al malestar psicológico determinado socialmente y su expresión por medio de actos violentos. El acercamiento metodológico de esta investigación es de tipo cualitativo y se trata de un estudio crítico con enfoque interpretativo. Participaron cinco hombres que se reúnen en un centro gubernamental especializado para la atención de la violencia familiar ubicado en una Delegación política del sur en el Distrito Federal. El malestar psicológico de la vida cotidiana se abordó a partir de instrumentos clínicos y del análisis de entrevistas grupales que exploran variables sociales. Además, se presentan los significados de los abusos realizados por los hombres con el fin de expresar el malestar. Se describió una lógica de acumulación de malestar con origen social expresado en un acto de abuso, y mediado por una razón o conflicto que justifica el ataque físico y/o verbal. La expresión de malestar, en ocasiones, se acompaña por sentimientos de culpa posteriores a los eventos violentos. Los elementos que permiten los abusos expresivos contra la pareja y otros familiares son las creencias sobre la superioridad del hombre, la inferioridad física de las mujeres e hijos, la propiedad de la persona por ser esposa, la obligación de la duración de una relación "para toda la vida" y las creencias sobre el uso de la violencia en las relaciones. En términos de intervención con esta población, se destaca la importancia de considerar el papel que juegan las determinantes sociales del malestar psicológico provenientes del nivel macro para los programas sociales e intervenciones comunitarias.

Palabras clave: Determinación social, malestar psicológico, hombres, violencia familiar, abuso.

\section{INTRODUCCIÓN}

Dentro de las interpretaciones que se pueden hacer sobre la violencia que ejercen los hombres contra sus parejas y otros familiares, destacan los principales elementos que dan origen a sus esquemas mentales, que se corresponden en mayor o menor medida con ideas estereotipadas sobre el género, la generación, las familias y las creencias sobre la validez del uso de la violencia. Estas creencias de género están presentes tanto en la denominada violencia instrumental como en la expresiva; pueden ser la variable principal en la génesis del malestar y en las motivaciones del acto, ${ }^{1,2} \mathrm{o}$ bien pueden permitir la expresión de malestar hacia parejas y otros familiares, no siendo excluyentes ambas posibilidades.

En los casos en que las creencias rígidas de género no son el principal determinante del malestar psicológico en los varones, se suele identificar al malestar que dirigen hacia sus parejas, como derivado de las frustraciones ambientales y el estrés social ${ }^{3}$ producto de factores presentes en los contextos sociales más cercanos, sin considerar la presencia de varia-

Universidad Autónoma Metropolitana.

Correspondencia: Fernando Bolaños Ceballos. La Fama 41, col. Industrial, Gustavo A. Madero, 07800, México, D.F. E-mail: hxefernando@gmail.com 
bles de la dimensión estructural. En este sentido, las respuestas sociales para enfrentar estos malestares o problemas de salud no suelen señalar las condiciones que operan a niveles más amplios para producir malestares psíquicos que se expresan en la violencia hacia la pareja y/o los familiares.

Algunos planteamientos del modelo explicativo del desarrollo humano de Bronfenbrenner ${ }^{4}$ son de gran utilidad para abordar las posibles variables estructurales que determinan el malestar psicológico individual. El modelo ecológico plantea la interacción de un sujeto activo en permanente desarrollo con distintos ambientes, entendidos como sistemas que tienen que ser estudiados para entender el desenvolvimiento de las personas, y que caracteriza al desarrollo como una progresiva acomodación mutua que también se ve afectada por las relaciones que se establecen entre los distintos entornos en los que participa la persona y los contextos más amplios en los que esos entornos están incluidos.

Este modelo concibe un conjunto de estructuras seriadas y estructuradas en diferentes niveles, en donde cada uno de ellos contiene al otro. El nivel más interno de estas estructuras lo forman los entornos inmediatos que contienen a la persona en desarrollo, llamados microsistemas (familia, escuela o trabajo, barrio). En el siguiente nivel se sitúan las relaciones entre dos o más entornos inmediatos de la persona en desarrollo y en los que actúa activamente, los que formarían el mesosistema. En el tercer nivel se sitúan los entornos donde la persona no está presente de forma activa, pero es influida por ellos: el nivel del exosistema. Y en el cuarto nivel se sitúan los factores socioeconómicos y culturales más amplios en los que se desenvuelven la persona y todos los individuos de su sociedad, lo que constituiría el macrosistema.

El presente artículo aborda variables que pueden estar operando en los diferentes sistemas, y particularmente las de origen cultural y estructural, para generar malestar emocional en hombres concretos que lo expresan en el abuso de la pareja $\mathrm{y} / \mathrm{u}$ otros integrantes de su familia.

Los varones que abusan en sus relaciones familiares han sido denominados, desde la perspectiva de género, como hombres que ejercen violencia masculina en el hogar. ${ }^{5}$ Con ello, se ha dado la connotación del acto violento (de los hombres) y su vínculo, con la dimensión social del mismo (violencia masculina). Así, se considera que cada hombre tiene una experiencia personal con respecto a la violencia que ejerce $\mathrm{y}$, al mismo tiempo, reproduce las estructuras de poder $\mathrm{y}$ subordinación dentro de la sociedad.

La violencia masculina se define como un acto que es simultáneamente instrumental y expresivo. Su instrumentalidad reside en que es un método de control social y, en ese sentido, es una estrategia de intimidación al servicio de la dominación, una estrategia que el hombre conscientemente elige desde su posición social. ${ }^{6}$ En el sistema patriarcal, los hombres están inscritos en sistemas sociales de poder que les brindan privilegios por el sólo hecho de ser varones, capital simbólico y real interiorizado que utilizan ante las crisis subjetivas relacionadas con los conflictos y las diferencias que ocurren en sus relaciones familiares. Es decir, la violencia guarda una importante relación con las crisis que atentan contra la identidad genérica o de "autoridad", ante las cuales se realizan las prácticas de abuso que mantienen el orden dominante de género. Por ello, el acto violento es un instrumento al servicio de cierta lógica y orden sociales.

En su sentido expresivo, la violencia puede ser entendida como una experiencia regresiva, relacionada con la historia de vida y experimentada como un sentimiento de "perderse", que se produce de forma paralela al sentido instrumental. La expresión del malestar psicológico que se realiza en el acto violento, presente antes o durante los conflictos de pareja y familiares, se dirige a expulsarlo del propio sujeto. ${ }^{6,7}$ Esta violencia expresiva (emocional, reactiva u hostil) se caracteriza por conductas violentas, realizadas impulsivamente y motivadas por sentimientos de ira y rabia, y usualmente se dirige hacia quienes tienen menos poder, es decir, hacia quienes se cree que son inferiores por razones de género o edad. 8,9 Esto ubica a la subjetividad relacionada con el género como la que direcciona su expresión hacia el espacio doméstico. Así, se pueden considerar los actos violentos en las relaciones familiares como una manifestación sintomática que sintetiza dos tensiones relacionadas con el malestar, una cultural-social y otra personal-psicológica.

Aunque el género resulta determinante en la génesis de la violencia familiar de los hombres, éstos presentan diferencias entre sí. Desde un enfoque psicológico, algunos autores han creado tipologías acerca de ellos, basados en dichas diferencias, por ejemplo, Dutton plantea tres tipos básicos: ${ }^{10}$ los que se caracterizan como psicopáticos y que no son candidatos a lograr cambios importantes en los programas de intervención; y los cíclicos o emocionalmente inestables que junto con los hipercontrolados, sí pueden lograr cambios en procesos reeducativos de tipo psicoterapéutico o reflexivo. Considerar esta tipología resulta indispensable para la reflexión sobre el carácter instrumental y expresivo de los actos violentos de estos dos últimos tipos, ya que podrá prevalecer en cierta medida el carácter instrumental (dominación por razones de género) sobre el expresivo (expresión de malestar personal), o viceversa, en los ejercicios de violencia, aunque siempre estén presentes los dos.

En el caso de los denominados hipercontrolados, la violencia de tipo instrumental es la que se presenta con mayor frecuencia, y es motivada por el malestar psicológico asociado al cumplimiento de creencias rígidas, entre ellas los estereotipos de género. En el caso de los emocionalmente inestables, el patrón de abuso responde más a la expresión del malestar, es decir, se relaciona con percepciones, emociones y circunstancias que ocurren con la pareja o familiares. Así, el carácter expresivo de la violencia como una experiencia regresiva, puede asociarse con experiencias de maltrato a temprana edad, así como de conflicto y violencia en la socialización y las relaciones adultas de pareja. En este caso se 
trata de un abordaje psicológico centrado en variables del nivel microsocial de la realidad.

Sin embargo, si se considera que la experiencia de cualquier sujeto y su grupo primario están contenidos en varios niveles de la realidad social, cabría preguntarse si es únicamente el malestar psicológico asociado con los sucesos ocurridos en la infancia lo que se manifiesta en los actos violentos expresivos. Al respecto, algunos estudios que han explorado el carácter expresivo de la violencia y lo relacionan con experiencias tempranas de maltrato por parte de los padres o cuidadores en hombres que ejercen violencia familiar, destacan que sólo alrededor de una tercera parte reporta experiencias de maltrato en la infancia. ${ }^{3,11,12}$ Es decir, aproximadamente el $70 \%$ de estos hombres expresa un malestar que no proviene de experiencias de maltrato en su infancia sino que es generado por otras razones, por ejemplo, las crisis producto del incumplimiento de las creencias rígidas sobre los roles de género, experiencias traumáticas producto de la socialización masculina o de otros eventos presentes en la trayectoria de vida. Pero también es necesario considerar otras determinantes sociales ${ }^{13}$ del malestar psicológico que pueden ser expresadas en los actos violentos, y que provienen de otros niveles de la realidad además de los niveles micro, meso y exo. Es decir, del contexto social, incluido en éste el nivel macro de la realidad.

Desde una mirada micro de la realidad, se plantea que la violencia expresiva refleja dificultades en el control de los impulsos y un estado emocional de activación fisiológica desagradable inducido por una frustración ambiental, o por una interpretación sesgada del comportamiento de los demás. ${ }^{14}$ En estos episodios del trastorno explosivo-intermitente, el grado de agresividad empleado es desproporcionado con respecto a la intensidad del estresor psicosocial que lo precipita (por ejemplo, un fracaso académico, una pérdida de trabajo, accidentes, etc.). El hombre, antes del comportamiento violento, puede sentir una gran tensión interior que posteriormente va seguida de una sensación de liberación, y más tarde por sentimientos de culpa y de arrepentimiento. Así suele comportarse "con normalidad" entre un episodio explosivo y otro, lo que hace pensar que estos ataques expresan un malestar producto de una condición psicofisiológica inducida por un estresor de origen social.

Estos estresores sociales, al no especificarse o ubicarse únicamente en el nivel meso o micro de la realidad, no identifican a otras determinantes sociales de los niveles exo y macro de la realidad que contribuyen a la generación de dicha afectación. Ante lo anterior, cabría preguntarse ¿Qué se puede entender por "frustración ambiental" o "estresor psicosocial"? Retomando factores del ámbito educativo, laboral y otras variables sociales la reflexión se orienta hacia las condiciones materiales de vida de los sujetos como generadoras del malestar psicológico y su relación con la violencia expresiva en el ámbito familiar.

A esta violencia se le denominará de aquí en adelante "abuso expresivo", en la medida en que son actos de violencia expresiva que ocurren en un vínculo de confianza, motivo por el que precisamente son abusivos, pues no se darían en otras relaciones. Son el síntoma de una serie de variables sociales filtradas, negociadas y reelaboradas por la experiencia personal de los sujetos y manifestada en su salud y sus prácticas sociales. Es decir, variables generadoras de malestar que se muestran como parte de un proceso de determinación de la salud y la enfermedad ${ }^{15}$ que va más allá de los niveles micro, meso y exo de la realidad social, y que incluye el nivel macro social (figura 1).

Con base en lo anterior, el objetivo de este artículo es conocer y describir la experiencia subjetiva de hombres que asisten a un programa reeducativo para detener su violencia familiar, en torno al malestar psicológico determinado socialmente y su expresión por medio de actos violentos. Es decir, se trata de describir el proceso de un síntoma personal y subjetivo, que, al mismo tiempo, es una condición social y estructural.

\section{MATERIAL Y MÉTODOS}

El acercamiento metodológico de esta investigación es de tipo cualitativo, y se trata de un estudio crítico con enfoque interpretativo. ${ }^{16}$ La determinación social de la salud y la enfermedad es el abordaje teórico que atraviesa la propuesta metodológica, que a su vez incorpora un enfoque de jerarquización de determinantes. Tiene un diseño no experimental, no hay asignación al azar, sino que se trata de una situación que previamente existía, ajena al control directo del investigador y donde los sujetos, por autoselección, ya pertenecían a un grupo o nivel determinado; es transversal,

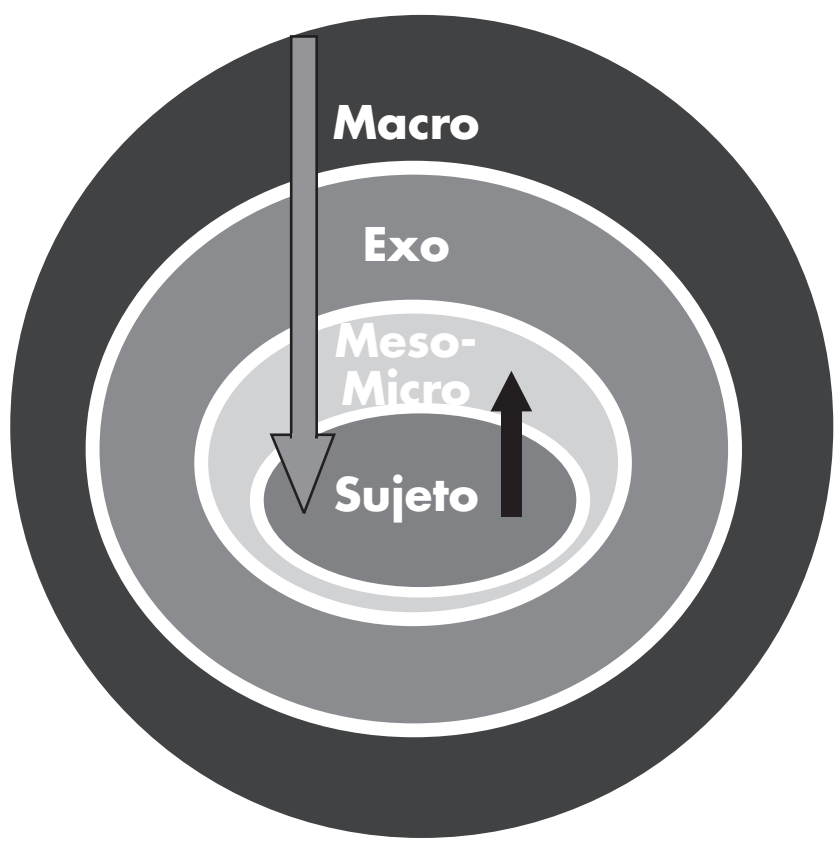

Figura 1. Determinación social multidimensional del malestar psicológico y el abuso expresivo 
ya que se centra en analizar datos recopilados en un punto en el tiempo. ${ }^{16}$ Los instrumentos se pilotearon en un grupo con similares características que acudía a otra institución, para detectar posibles dificultades de diseño o en la aplicación y ajustarlos.

Participaron cinco hombres que se reúnen en un centro gubernamental especializado para la atención de la violencia familiar ubicado en una Delegación política en el sur del Distrito Federal, limítrofe con el Estado de Morelos, por lo que se puede caracterizar a este grupo como semirural. Sus principales características se describen en el cuadro 1.

Todos respondieron los instrumentos autoaplicados y participaron en una entrevista grupal guiada temáticamente para abordar los factores sociales que les generan malestar, las manifestaciones de éste y los significados detrás de los abusos expresivos. Los instrumentos individuales se respondieron antes de la entrevista, con el fin de recopilar información sobre posibles problemas que representarán determinantes sociales del malestar, así como síntomas de estrés. Se utilizaron dos inventarios validados en México denominados Lista de problemas de Mc Kay, Davis y Fanning, ${ }^{17}$ cuya finalidad es la identificación de áreas problemáticas para los sujetos y que consta de 80 situaciones o problemas divididos en nueve áreas (salud, economía, trabajo, vivienda, relaciones sociales, ocio, familia, psicológica y otros.) y la escala sobre sintomas de estrés de Reynoso et al., que en su primera parte indaga sobre la presencia o ausencia de 14 problemas o enfermedades consecuencia del estrés. En la segunda parte se presenta un listado de 23 síntomas de estrés buscando que el sujeto responda sobre la presencia o ausencia de éstos. ${ }^{17}$ Cada área de la lista de problemas se calificó a partir de la frecuencia en las respuestas positivas y el grado de molestia señalado; la escala para el estrés se calificó a partir de las respuestas positivas de los sujetos. Además con base al formato de preingreso al grupo, elaborado por Bolaños para el "Programa multidimensional y multicomponente para detener la violencia de género de los hombres hacia sus familiares", ${ }^{18}$ se aplicó una escala adaptada para ubicar a agresores la tipología de Dutton, ${ }^{10}$ algunos reactivos sobre antecedentes de maltrato infantil y otros que exploraron el nivel de ingresos y escolaridad para determinar las condiciones económicas generales de los participantes. Los instrumentos fueron calificados manualmente y se sintetizó la información contenida en cada apartado; la escala para agresores se calificó a partir de criterios preestablecidos para la adaptación.

Las entrevistas grupales fueron grabadas con el consentimiento del grupo y transcritas con un procesador de palabras, procesadas con el softwere ATLAS TI y analizadas a partir de un enfoque interpretativo. Se exponen las narraciones del grupo respecto a la relación entre variables sociales y el malestar psicológico que experimentan en las áreas: trabajo, salud, apoyos gubernamentales, redes sociales, cambios en las relaciones de género y otras identificadas por ellos mismos. Posteriormente, se describen los ejercicios de abuso expresivo y los elementos que les permiten dirigir la expresión del malestar hacia la pareja e hijos/as.

\section{RESULTADOS}

Las áreas que resultaron más problemáticas para estos hombres, según la lista de problemas son: la familiar, la económica, la psicológica y la salud. Los principales síntomas de estrés que reportaron son: dolores musculares, insomnio, pesadillas y cambio en las conductas habituales. Las respuestas positivas del grupo a los síntomas de estrés considerados en todo el instrumento representan el $43 \%$ de los reactivos.

Sobre los antecedentes personales de violencia, destaca que todos reportaron haber sido testigos de violencia emocional y verbal contra su madre por parte de su padre o pareja de la madre, y cuatro de violencia física. Tres observaron violencia verbal por parte de su madre hacia el padre o pareja de la madre, y uno identifica a la madre o pareja del padre como agresora física. Sobre la violencia directa, todos reportaron haber recibido la verbal y emocional por parte de sus familiares y tres reportaron violencia física; en ambos casos, destaca la figura paterna como el principal agresor. Respecto al maltrato durante la infancia que les resultó más impactante o doloroso y quién lo realizó, reportaron el proveniente de los padres y madres o figuras sustitutas, y las formas fueron insultos y gritos hacia ellos y golpes entre adultos. No se reportó violencia en espacios sociales.

Tres de los cinco hombres se consideran dentro del perfil básico del maltratador, uno dentro del perfil de agresor hipercontrolado y uno más con rasgos psicopáticos. Destaca éste último porque se corresponde con el sujeto que punteó más alto en varias áreas de la lista de problemas y en la de síntomas de estrés.

Cuadro 1.

\begin{tabular}{ccccll}
\hline Seudónimo & Edad & Estado civil & Hijos & Escolaridad & Ocupación \\
\hline T1 & 48 & Divorciado & Sí & Preparatoria & Oficios \\
T2 & 43 & Casado & Sí & Carrera técnica & Comerciante \\
T3 & 25 & Soltero & No & Preparatoria & Desempleado \\
T4 & 20 & Soltero & No & Preparatoria incompleta & Estudiante \\
T5 & 47 & Separado & No & Preparatoria incompleta & Comerciante \\
\hline
\end{tabular}




\section{El discurso grupal sobre la determinación social del malestar}

Trabajo remunerado

Se señala que el estrés ocurre por las condiciones actuales del trabajo remunerado y el nivel de vida familiar que tienen en comparación con años anteriores; otros, mencionan que sólo cuando no tienen trabajo remunerado; y otro más, por el presente y el futuro de tal condición pero asociada principalmente al temor de incumplir con las expectativas tradicionales sobre el rol de hombre-padre-proveedor. Se podría decir, en términos del malestar, que para algunos hombres se destaca un antes y un después ante las condiciones de esta esfera, además de la posibilidad de su ausencia en el presente y en ciertos escenarios futuros.

\section{Salud}

La relación entre la insatisfacción de sus necesidades por el sistema de salud y el enojo, impotencia y estrés que ello genera, presenta una cierta normalización que consideran imposible de modificar. Los servicios se utilizan regularmente, excepto en el caso de situaciones de salud consideradas de relevancia, en las que se acude a un servicio privado. Consideran que la mala atención que recibe quién la solicita afecta las actividades remuneradas de los familiares generando estrés, ya que tienen que prestar apoyo o compañía para conseguir satisfacer su demanda de salud.

\section{Apoyos gubernamentales}

Sobre la relación entre el funcionamiento de los programas gubernamentales federales o locales, o la falta de ellos, este grupo de hombres manifestó un importante malestar. También reportan como un estresor social la presencia de información gubernamental en los medios masivos de comunicación que indica mejoras en las condiciones de vida pero no percibirlas en su experiencia cotidiana, además de un efecto de "engaño" en "las personas", asunto que consideran presente desde administraciones pasadas. Emergió el tema del crimen organizado y la violencia que afecta al país como un fenómeno que también les genera malestar en forma de temor y frustración; este malestar se asocia con la falta de inversión de su capital para mejorar el negocio propio o sus bienes materiales. Finalmente, se identificó a los funcionarios públicos locales como otras variables sociales generadoras de estrés, al ejercer extorsión y corrupción que les afecta como comerciantes.

\section{Redes sociales}

Respecto de las redes sociales, las narraciones se dirigieron hacia la ausencia de apoyos públicos a las necesidades básicas, reportando malestar al identificar su presencia en un pasado relativamente reciente. Otras frustraciones ambientales se refieren a la falta de apoyos para la producción agrícola en estas zonas y a la corrupción de ser- vidores públicos, además de considerar que el Estado no cumple con su función de mejorar las condiciones de vida "del pueblo".

\author{
Cambios en las relaciones \\ de género y apoyos a mujeres
}

Respecto de los estresores asociados a los cambios en las relaciones de género y la existencia de apoyos públicos dirigidos a mujeres, sólo un hombre reportó como estresante un programa público para mujeres que quieren estudiar con el fin de detonar su movilidad laboral, lo que generó que su esposa percibiera mayores ingresos y él entrara en una crisis, así como su relación de pareja en general. El apoyo hacia la mujer se consideró una situación injusta que influyó en que el hombre no cumpliera con el papel tradicional de proveedor.

\section{Otras variables sociales}

Los estresores sociales que consideraron los entrevistados como no abordados en la guía de entrevista fueron: el temor a ser víctima de un delito o del crimen organizado; modificación de sus dinámicas de vida debido a la inseguridad y su efecto en limitar la generación de recursos económicos; la corrupción de servidores públicos que afecta los comercios; las dificultades para el pago de los impuestos; el aumento de competencia en el comercio y su asociación con generar menos ingresos; la falta de trabajo que impide el cumplimiento del papel como proveedor; considerar que el trabajo es la mayor de las frustraciones que hay que superar en la vida; el mal uso del dinero para la pensión alimenticia de una menor por parte de la ex-pareja; la falta de reparación del pavimentado de calles, y considerar que se usan los recursos para beneficiar a personas que apoyan a un partido político, y, finalmente, el pago de cuotas en las escuelas y guarderías públicas.

\section{El abuso expresivo en la violencia familiar}

Se reportó que los estresores sociales y las frustraciones ambientales mencionadas se convierten en un malestar latente ante el cual se busca el detonante para ejercer violencia, citando, como ejemplo, los reclamos por parte de la pareja o los avisos de mala conducta de los/as hijos/as: "ya llevas problemas del trabajo... ya llevas la energía suficiente para que desates una guerra", ya que "...es fácil encontrar un detonante que dispare todo eso que ya está en una olla express", porque "una plática la hacemos una discusión", "pero como ya traes la carga completa, ya dices 'pues aquí"'; y por lo tanto, "...ahí como que se descarga". Otros abusos expresivos se detonan ante la falta de argumentos de discusión durante los conflictos, por ejemplo: "...como ya no tengo nada que decirte, entonces te voy a echar todo lo que traigo". Finalmente, algún hombre comentó: "Sí lo saca uno, pero ya después queda como el remordimiento, la culpa de 
que por qué hiciste las cosas de esa manera cuando no tenía que ser así". Otros hombres expresan este malestar "entreteniéndose con algo", e identifican que, como "la tensión se queda ahí", ejercen violencia verbal.

\section{Los elementos que permiten el abuso expresivo hacia familiares}

Además de las creencias sobre el uso de la violencia, las variables que permiten el abuso expresivo contra la pareja y otros familiares son las creencias dominantes de género, de generación y sobre las relaciones de pareja, como se observa en los testimonios del cuadro 2.

Estas creencias las identificaron dentro de una lógica general de relaciones de poder en los espacios sociales, laborales y familiares. Manifestaron que está presente una actitud constante "a la defensiva", y cuando se recibe un abuso por alguien con más poder, se expresa el malestar mediante la misma dinámica: "a lo mejor no puedo hacerte porque estás grandote, pero más adelante me encuentro un chaparrito y hasta le doy una patada...". En este sentido, la expresión del malestar se dirige hacia alguien "inferior", no forzosamente por talla física, sino por el tipo de relación de poder, por ejemplo "... [ante un evento de violencia con el empleador], pero vas a perder el trabajo ¿sí? Yo sé que si me peleo con mi patrón va a decir 'mañana, aquí mismo se termina tu trabajo y ahí te ves y te pago lo que hiciste ${ }^{\prime \prime \prime}$.

Cuadro 2. Creencias que permiten el abuso expresivo

"Es que ahí estás viendo la diferencia de debilidades, realmente lo hacemos porque ellos son... más frágiles... no lo vas a hacer con alguien que te va a devolver tal por cual $\dot{\partial}^{\text {no? }}$. Desgraciadamente, ellos son los que están expuestos porque... ella como mujer es más débil que uno, los niños pues por superioridad, tanto ellos son pequeños... desgraciadamente pues así lo hacemos...".

"Pero no me desahogué con él y era el problema con él [el empleador], sino llegar a casa y desquitarme con los demás, pero como los vemos débiles, los vemos como que, 'yo soy el superior, y aquí se tiene que tener respeto y aquí se tiene que hacer lo que yo digo'. Ahí, entonces ¿qué se hace? 'Pues ni modo, es el papá y lo tienes que...' y ipum! ahí se cae...".

"Porque yo sabía que ella era mi esposa y siempre iba a vivir conmigo hasta que yo me muriera o ella se muriera, vivíamos con esa... ella es mía y nunca me va a dejar ¿sí? Entonces por eso decía yo, 'y aquí vengo y descargo', 'y aquí vengo y hago porque de todos modos es mía'. Más sin en cambio, en mi trabajo lo voy a perder, ahí no me puedo desquitar, mejor me desquito acá, que puedo vivir con ella hasta... mañana le pido una disculpa y ya se acabó el problema y ya no pasa nada. Así como yo llegaba y descargaba, así ella llegaba y todo lo cargaba".

"Y yo sé que a lo mejor dices... 'pero me va a dejar mi mujer', y si ya lo vemos de esa manera, ya ni le hacemos el daño a ella ${ }_{\partial}$ sí? Yo nunca lo pensé de esa manera, pero si yo sabía que ese era el problema, o si ese iba a ser el problema de una separación con mi pareja, yo la cuido".

\section{DISCUSIÓN}

Los principales problemas de estos hombres tienen que ver con las esferas de la familia y la economía (que provienen principalmente de los niveles macro y exo), además de las áreas que afectan la salud mental y física, teniendo para esta última la presencia global de cerca de la mitad de los síntomas de estrés explorados. Las principales esferas generadoras de malestar provienen de todos los niveles de la realidad, resultando únicamente los problemas familiares parte del perfil específico de esta población.

Los estresores sociales o frustraciones ambientales que se destacaron en las entrevistas debido a su densidad en las narraciones fueron: el desempeño gubernamental en términos de falta de opciones de empleo o empleos dignos, la desaparición de programas sociales y la violencia criminal en todo el país; el mal desempeño gubernamental local en términos del funcionamiento de los programas sociales, la corrupción y su relación con la economía personal y familiar, y la delincuencia.

Debido a la alta presencia de experiencias violentas en sus familias de origen, se puede considerar que estos hombres están reproduciendo abusos experimentados en la infancia, pero además se observó la expresión de malestar emocional determinado socialmente en sus actos violentos. Aunado a ello, a la mayoría se les consideró agresores emocionalmente inestables, y en menor medida, como hipercontrolados o con rasgos psicopáticos.

Al explorar la relación entre el malestar emocional con el origen social y los actos de abuso, así como para identificar las creencias que permiten los abusos expresivos y la experiencia de estos actos, resultó necesaria la reiteración de la pregunta en más de dos ocasiones. Se considera que los significados sociales y personales sobre estas relaciones son elementos que necesitan construirse para los sujetos, ya que se encuentran invisibilizadas o naturalizadas en sus esquemas mentales.

Se describió una lógica de acumulación de malestar con origen social expresado en un acto de abuso y mediado por una razón o conflicto que justifica el ataque físico y/o verbal. La expresión o "descarga" de malestar en ocasiones se acompaña por sentimientos de culpa posteriores a los eventos violentos. Los elementos que permiten los abusos expresivos contra la pareja y otros familiares son las creencias sobre la superioridad del hombre, la inferioridad física de las mujeres e hijos/as o la creencia sobre ello, la propiedad de la persona por ser esposa, la obligación de la duración de una relación "para toda la vida", y las creencias sobre el uso de la violencia en las relaciones. Todo lo anterior forma parte de una lógica social de relaciones de poder, donde se reproducen estos actos ante la inferioridad real o simbólica de las personas.

Al considerar que los estresores sociales y las frustraciones ambientales son determinadas socialmente por va- 
riables que proceden de todos los niveles de la realidad, incluido el macrosocial, identificamos el papel que puede jugar la estructura social en la génesis del malestar que se expresa en abusos. Así mismo, estos actos expresivos de malestar son dirigidos hacia las mujeres y niños/as que forman parte de vínculos familiares debido a elementos culturales provenientes del nivel macro, pero que atraviesan todo el ambiente de los sujetos. Así, se describe la interacción de un sujeto activo en permanente desarrollo con su ambiente multidimensional, en este caso, en términos del malestar psicológico determinado socialmente y las prácticas de abuso expresivo hacia familiares.

En términos de intervención con esta población, se destaca la importancia del papel que juegan las determinantes sociales del malestar psicológico provenientes del nivel macro para los programas sociales e intervenciones comunitarias. Enfatizamos que la sola reeducación en género de los hombres agresores, aunque es muy importante, únicamente modifica la direccionalidad de la expresión de ese tipo de malestar, y no disminuye las causas que le dan origen, lo cual resulta en acciones que sólo administran un problema de salud sin buscar su solución. De forma paralela, se debe considerar que mientras exista mayor estrés y frustración en las condiciones de vida de los sujetos, será más difícil pensar en acciones de prevención primaria, secundaria o terciaria exitosas, hablando de temas que implican la reeducación de género en salud u otros de interés, ya que estas problemáticas tendrían que ser consideradas como prioritarias por los propios sujetos, frente a la insatisfacción de sus necesidades básicas.

En términos de investigación, cabría preguntarse cuáles son las determinantes sociales del malestar psicológico de los diferentes grupos de hombres que no ejercen violencia familiar, ya que para esta población resulta una característica importante de su propio perfil. Abordar esta problemática en poblaciones representativas y con estudios cualitativos, permitiría identificar las determinantes sociales de la saludenfermedad de los varones, sus problemáticas asociadas y la propia experiencia de los hombres en su contexto amplio; en esa medida, se contribuirá a generar un conocimiento que potencialice las acciones públicas que brindan atención a los hombres en sus diferentes niveles.

\section{REFERENCIAS}

1. Montoya O. Nadando contra corriente. Buscando pistas para prevenir la violencia masculina en la relación de pareja. Nicaragua: Puntos de Encuentro; 1998.

2. Batres G. El lado oculto de la masculinidad. Tratamiento para ofensores. San José de Costa Rica; ILANUD, Programa Regional de capacitación contra la Violencia Doméstica; 1999.

3. Farnós de los Santos T. Las raíces psicosociales y culturales de la violencia. Documentación social 2003;(131):11-30.

4. Bronfenbrenner U. La ecología del desarrollo humano. Barcelona: Ediciones Paidós; 1987.

5. Kaufman M. Las experiencias de poder contradictorias entre los hombres. Masculinidades. Poder y crisis; Santiago de Chile: Isis Internacional; 1997.

6. Goldner V. Love and violence: Gender paradoxes in volatile attachments. Family Process 1990;29(4):333-364.

7. González L, Villacorta C. Aproximación teórica a la Violencia; San Salvador; 1998; http://www.uca.edu.sv/publica/eca/599art4.html. Consulta: dos de marzo de 2013.

8. Scott J. El género como una categoría útil para el análisis histórico. En: James SA, Mary JN (eds.). Historia y género: las mujeres en la Europa moderna y contemporánea. España: Universidad de Valencia; 1990; pp. 23-58.

9. Connell RW. Masculinidades. México: PUEG/UNAM; 2003.

10. Dutton D. El golpeador. México: Ed. Paidós; 1997.

11. Echeburúa E, Fernández-Montalvo J, Amor PJ. Psychopathological profile of men convicted of gender violence: A study in the prisons of Spain. J Interpersonal Violence 2003;18:798-812.

12. Fernández-Montalvo J, Echeburúa E, Amor PJ. Aggressors against women in prison and in community: An exploratory study of a differential profile. International J Offender Therapy Comparative Criminology 2005;49:158-167.

13. Iriart C, Waitzkin H, Breilh J, Estrada A et al. Medicina social latinoamericana: aportes y desafíos. Temas de actualidad. Rev Panam Salud Publica 2002;12(2):130-132.

14. Amor P. Personalidades violentas. 2007; http://www.revista-critica. com/articulos.php?id=1091; Consulta: 12 febrero 2013.

15. Castro R. Teoría social y salud. CRIM-UNAM; Buenos Aires: Lugar editorial; 2011.

16. Hernández S. Fernández C. Baptista P. Metodología de la Investigación. Segunda Ed; México: Mac Graw Hill; 1998.

17. Reynoso L, Seligson I. Psicología clínica de la salud: un enfoque conductual. Instituto de Neurociencias de la Universidad de Guadalajara y Facultad de Psicología de la Universidad Nacional Autónoma de México. México: Manual Moderno; 2005.

18. Garda R, Bolaños F. Caminado hacia la equidad. Programa multidimensional y multicomponente para detener la violencia de género de los hombres hacia sus familiares. México: Instituto Nacional de las Mujeres-Hombres por la Equidad A; 2010.

Artículo sin conflicto de intereses 